Background and Aim The aim of this study, the frequency of catheter associated blood stream infection (CA-BSI) and its effect to mortality and length stay (LOS) of hospital.

Methods This study was conducted between November 1, 2010 and February 29 2012, and it is prospective and observational.

Results During study period, 275 patients admitted to PICU. Fifty-six percent of all patients were girl and their mean age were $87 \pm 87.4$ months. There was CVC in $107(38.9 \%)$ patients. Also, there were CVC at vena jugularis interna (VJI) in $48.9 \%$, femoral in $46.7 \%$ and subclavian in $4.3 \%$ of patients with CVC. There were 23 times CA-BSI in 16 (14.8\%) patients. Totaly CVC use day was 1589 days and CA-BSI was 14 attack/1000 days within study period. The agents of CA-BSI were A. Baumannii (26\%), MR-Coagulase Neagative Staphylococcus (21.7\%), ESBL (+) Kl. Pneumonia (21.7\%), VRE (8.6\%), P. Aeruginosa (8.6\%). There were 169 patients without CVC and $4(2.4 \%)$ of them BSI. CA-BSI weas $85 \%$ of all BSI. The LOS of PICU was $43.7 \pm 63.7$ days in patients with CA-BSI and $11 \pm 11.4$ days in patients without CA-BSI in patients with CVC $(p=0.005)$. The LOS of PICU in patients without CVC; $29.7 \pm 16.1$ days in BSI group and $5.1 \pm 5.7$ days in without BSI group $(p=0.001)$. During study period, $36(13 \%)$ patients died and 5 of them were related CA-BSI.

Conclusion CVC use is severe risk factor for CA-BSI, LOS of PICU and mortality.

\section{CASE-CONTROL ANALYSIS OF ENDEMIC ACINETOBACTER BAUMANNII BACTEREMIA IN THE NEONATAL INTENSIVE CARE UNIT}

doi:10.1136/archdischild-2012-302724.0585

JF Hsu, MH Tsai, SM Chu, R Lien, MC Chiang. Chang Gung Memorial Hospital, Chang Gung University College of Medicine, Taoyuan, Taiwan R.O.C.

Background and Aim Acinetobacter baumannii has become an important cause of nosocomial infection, but little is known about its impact on the neonatal intensive care unit (NICU). We planned to characterize the clinical manifestations and outcomes of patients with A. baumannii bacteremia in the NICU.

Methods All patients with A. baumannii bacteremia in our NICU from 2003-2010 were reviewed. A matched case-control study was performed by comparing each case of $A$. baumannii to 2 uninfected controls and all cases of Escherichia coli and Klebsiella bacteremia, respectively.

Results 37 sporadic cases of A. baumannii bacteremia were identified. Pan-drug resistant isolate was noted in only 2 cases (5.4\%), and the overall mortality rate was $8.1 \%$. Infants with $\mathrm{A}$. baumannii bacteremia had median gestational age and birth weight of 28 weeks and 1090 grams, respectively. Compared to matched, uninfected controls, infants with A. baumannii were more likely to have had a central vascular catheter (CVC) (OR=3.78; 95\%CI: 1.44 to 12.35 ) and longer duration of ventilator use and hospitalization (both $\mathrm{P}<0.001)$. Compared to E coli or Klebsiella bacteremia, infants with A. baumannii bacteremia had lower birth weight (median of $1090 \mathrm{gms}$ vs $1300 \mathrm{gms}, \mathrm{P}=0.044$ ) and a higher rate of CVC and TPN use (both $\mathrm{P}<0.001$ ) at the time of infection.

Conclusions A. baumannii bacteremia occurs sporadically in the NICU, primarily in low birth weight infants on TPN use and with CVC in situ. Although A. baumannii does not often cause mortality and PDR- A. baumannii is uncommon, it contributes significantly to longer hospitalization.
J Smisek, V Fiala, R Plavka. Division of Neonatology, Department of Obstetrics and Gynecology, General Faculty Hospital, Prague, Czech Republic

Background and Aims Coagulase negative Staphylococci (CoNS) are most prevalent pathogens in central line associated bloodstream infections (CLABSI) in very low birth weight (VLBW) infants. The aim of this study was to compare CLABSI caused by CoNS in terms of virulence and clinical relevance.

Methods A retrospective observation analysis of all CLABSI caused by CoNS in VLBW infants admitted to our NICU during a 5 -year period (2006-2010) was performed. Two groups of CLABSI were compared: the OXAS caused by CoNS susceptible to oxacillin and the OXAR caused by CoNS resistant to oxacillin, in terms of perinatal demographic data, related laboratory signs and clinical data.

Results There were 54 episodes of CLABSI caused by CoNS found in 51 infants, 14 in the OXAS group (average BW \pm SD: $855 \mathrm{~g} \pm 293$; average GA \pm SD: $25.9 \mathrm{wks} \pm 2.8$ ) and 40 in the OXAR group (average BW \pm SD: $788 \mathrm{~g} \pm 241$; average GA \pm SD: 26.2 wks \pm 2.3 ). The OXAR group presented a higher maximum CRP levels (median $\pm 95 \% \mathrm{CL}$ : $28 \pm 15 \mathrm{mg} / \mathrm{l}$ vs. $21 \pm 12 \mathrm{mg} / \mathrm{l}, \mathrm{p}=0.047$ ), as well as the maximum values of the I/T index (median $\pm 95 \% C L$ : $0.23 \pm 0.04$ vs. $0.19 \pm 0.05$, $\mathrm{p}=0.051$ ), higher number of positive blood cultures (median $\pm 95 \% \mathrm{CL}$ : $1 \pm 0.14$ vs. $2 \pm 0.3, p=0,006$ ) and the tendency to a higher incidence of necrotizing enterocolitis ( $38 \%$ vs. 14\%, p=0.078).

Conclusions Resistance to oxacillin in CoNS CLABSI has a relevant influence on higher levels of inflammatory markers and the tendency to NEC in VLBW infants.

\section{CAVITATING PULMONARY TUBERCULOSIS IN CHILDREN}

doi:10.1136/archdischild-2012-302724.0587

${ }^{1} \mathrm{G}$ şensoy, ${ }^{2} \mathrm{M}$ Ceyhan, ${ }^{1} \mathrm{~N}$ Belet, ${ }^{1} \mathrm{~A}$ Karlı. ${ }^{1}$ Pediatric Infectious Diseases; ${ }^{2}$ Radiology, Ondokuz Mayis University, Samsun, Turkey

Background and Aim To evaluate the clinical characteristics of children with cavitating pulmonary tuberculosis (PTB).

Patients and methods: We reviewed retrospectively 33 children followed at our hospital with a diagnosis of PTB between April 2007March 2012 and evaluated the clinical characteristics of patients with cavitary lesions. Tuberculosis (TB) was confirmed by isolation of Mycobacterium tuberculosis (M. tuberculosis) from early morning gastric aspirates, by positive tuberculin skin test (TST), history of exposure to tuberculosis, and/or detection of acid-fast bacilli (AFB) in the early morning gastric aspirates.

Results Seven (21\%) of 33 children had cavitating PTB. Median age was 13 years (range $0.4-15.5$ ), with a female/male ratio of $2.5 / 1$. All of them except one patient were $\geq 12$ years old. Three $(43 \%)$ patients had chronic cough ( $>4$ weeks) and two patients had hemoptysis ( 1 of them had massive hemoptysis). Contact with a case of TB in the family was available in all of the patients and TST was positive again in $100 \%$ of patients. M. tuberculosis was isolated in five $(70 \%)$ cases. AFB smear positivity was detected in three patients. Chest $\mathrm{X}$-ray and/or thorax computed tomography revealed consolidation in six cases (upper lob involvement in three cases), hilar lymphadenopathy in three and multiple cavities in two cases.

Conclusion Cavitating pulmonary tuberculosis is rare in childhood, but it can be seen especially in older ages. It should be kept on mind that adolescents with cavitary lesions can be potentially contagious and they should be treated with infection control measures. 